\title{
Comparative analysis of the pathogenic effect of the association of eimeria on hybrid rabbits at different doses of infection
}

\author{
$K$. Sidorenko, $M$. Mkrtchyan*, and $O$. Kaidalova \\ FSBEI HE “Saint-Petersburg State University of Veteriny Medicine”, Saint-Petersburg, Russia
}

\begin{abstract}
Rabbit breeding is one of the promising branches of animal husbandry, but its development is hampered by various diseases. The problem of the pathogenic influence of parasites of the genus Eimeria on the organism of rabbits at different infective doses is still quite urgent. The study was conducted on hybrids of rabbits of Soviet Chinchilla and Californian breeds at 30 and 45 days of age. Within the framework of the experiment 6 groups of young rabbits were formed by the method of pair-analogs. The one-day old rabbits in the experimental groups were infected with oocysts of the association of Eimeria species E. perforans and E. irresidua at doses of 100000 (2 groups) and 200000 (2 groups) per head, and in control groups ( 2 groups) - remained intact throughout the experiment. To analyze the pathogenic effect of parasites determination of meat productivity, veterinary and sanitary assessment of carcasses, linear measurements of intestinal length as well as histological studies of the walls of the small intestine were carried out in rabbits of these age groups. The research results showed that rabbits-hybrids infected at a dose of 100000 and 200000 oocysts per head demonstrate significant reduction of the increase in live weight and slaughter yield, despite the preservation of the retail display of the carcasses.
\end{abstract}

\section{Introduction}

Rabbits are herbivores with high fertility, excellent growth rates and feed conversion efficiency, playing an important role in solving the problem of protein deficiency in many parts of the world. All this makes rabbit breeding one of the most promising and fast-growing branches of animal husbandry [1].

In the market economy conditions there is an increased demand for rabbits as a source of meat, fur as well as using them for various experimental studies [2].

Thus, rabbit-breeders and rabbit-caretakers must have knowledge of the most dangerous diseases of rabbits in order to avoid economic losses, zoonotic threats and provide a diseasefree animal model for various scientific research. This article is aimed to provide insight into eimeriosis - the most common invasive disease of rabbits [3]. Representatives of the genus Eimeria are strictly specific, and under natural invasion no species infects more than one host. The Eimeria species parasitizing in rabbits undergo a

${ }^{*}$ Corresponding author: laulilitik@yandex.ru 
complex life cycle, including both intracellular and extracellular stages, as well as asexual and sexual reproduction [4].

Rabbits become infected with coccidiosis by ingesting sporulated oocysts and the invasion develops into a disease [5].

Young rabbits are more susceptible to infection, while adults are mainly parasitic carriers. The commonly observed clinical signs are: anorexia, a decrease in live weight and carcass weight, mainly caused by a decrease in feed intake, water and electrolyte imbalance in the affected part of the intestine accompanied by a loss of water and sodium, compensated by the potassium exchange from the blood, which leads to hypokalemia and death of an animal $[6,7,8]$.

All types of eimeria that affect the intestinal tract multiply in the epithelial lamina of the mucous membrane. Clinical signs vary and are most pronounced in young animals. There is a decrease in live weight gain, diarrhea (up to hemorrhagic), polydipsia, and sometimes death. Liquid intestinal contents, a contaminated area around the anus are often observed at a sufficiently high intensity of invasion. More mature animals can secrete eimeria oocysts even in the absence of clinical signs of the disease $[9,10]$.

The aim of our research is a comparative analysis of the pathogenic effect of different doses of infection with protozoa of the genus Eimeria on different age groups of hybrid animals of the meat-fur rabbit breed Soviet Chinchilla with the meat rabbit breed California.

\section{Materials and Methods}

The studies were carried out on the basis of the Department of Biology, Ecology and Histology of St. Petersburg University of Veterinary Medicine.

The study was conducted on hybrids of rabbits of Soviet Chinchilla and Californian breeds.

Groups of 6 heads in each were formed by the method of analogous pairs. The one-day old rabbits in the experimental groups were infected with oocysts of the association of Eimeria species E. perforans and E. irresidua at doses of 100000 (2 groups) and 200000 (2 groups) per head, and in control groups (2 groups) - remained intact throughout the

experiment. The animals were kept in a wall-mounted brood-feeding cage "Industrial Version 2.0" with bunker feeders and nipple drinkers.

The rabbits received feed of the "Mister Rabbit" PK 90-1 line, produced by the Tosno Mixed Feed Factory. Water was given without restrictions.

To study the pathogenic effect of these protozoa on the body of 30- and 45-day-old rabbits the live weight, slaughter weight and slaughter yield of carcasses were determined, a complete parasitological dissection of the gastrointestinal tract was carried out, linear measurements of the length of the small and large intestine, as well as veterinary-sanitary assessment of slaughter products(carcasses ) were conducted.

The rabbits were weighed using scales SUPRA BSS-4085 on the first day after birth, as well as before and after slaughter.

On the 30th and 45th day of infection, the animals of the experimental and control groups were slaughtered.

Linear measurements of the small and large intestines were also performed. First, the small and large intestine was freed from the mesentery and fat, after which it was straightened and linear measurements were made.

Statistical processing of the obtained data was carried out in the Past3 program. The Mann-Whitney test was used as a validation check.

Organoleptic assessment of carcasses, pathological and parasitological studies were carried out according to the generally accepted method. 
Sampling for histological examination (sections of the small and large intestines) measuring $10 \mathrm{~mm} \times 10 \mathrm{~mm}$ was carried out with sharpened eye scissors. Samples were signed, placed in histological cassettes and immersed in standardized buffered $10 \%$ formalin for 2 to 4 days for fixation. Before passing through series of alcohol, the specimens of organs were washed in running tap water for 1 - 2 hours. After that they passed through a six-stage processing in isoprep (based on isopropyl alcohol) of the same grade $(100 \%)$ for 20 minutes as well as three-stage imbedding in HISTOMIX paraffin for 20 minutes to form blocks. 4 $4.5 \mu \mathrm{m}$ thick sections were made on a rotary motorized microtome ROTMIK-2M. They were dewaxed in xylene and $96 \%$ ethanol (4 minutes in each), followed by immersing in distilled water for 5 minutes.

Staining was performed with alcian blue and Carazzi's hematoxylin according to the following protocol:

- $\quad$ Staining with alcian blue for 30 minutes.

- $\quad$ Drying of the section without rinsing using filter paper or tissue.

- Differentiating in $96 \%$ ethanol for about 5 seconds and washing in distilled water.

- Staining with Carazzi's hematoxylin for 45 seconds followed by dehydration and clarifying in xylene and $96 \%$ ethanol (4 minutes in each), after which they were placed under a synthetic mounting medium.

The finished preparations were examined at $\times 400$ and $\times 1000$ magnification.

The reported study was funded by RFBR, project number 19-316-90059.

\section{Results and Discussion}

No clinical signs were found in the rabbits of the experimental groups with the intensity of the invasion of both 100000 and 200000 within 30 and 45 days after infection.

The results of studies of the pathogenic effect of eimeria on weight gain and slaughter yield of meat, depending on the dose of infection in one-month-old animals, are shown in Figure 1.

As can be seen from the data in Figure 1, the animals of the control group showed the best results in average live weight, slaughter weight and slaughter yield of carcasses. At the same time, the largest gap in live weight is observed between intact rabbits and infected with 200000 oocysts slaughted at 30 days of age.

The difference was $35.6 \%$, which means more than a third of the lost live weight. A similar indicator for infection with 100000 is less than control by $16.4 \%$, which is also very significant. Slaughter yield in both experimental groups showed a $3 \%$ decrease in relation to the intact group. The contamination dose of 200000 oocysts most significantly affected the slaughter weight of the carcass, which was $261.37 \mathrm{~g}$ (39\%) less than in the control group and $122.5 \mathrm{~g}$ less than in rabbits that received a lower dose of oocysts. 


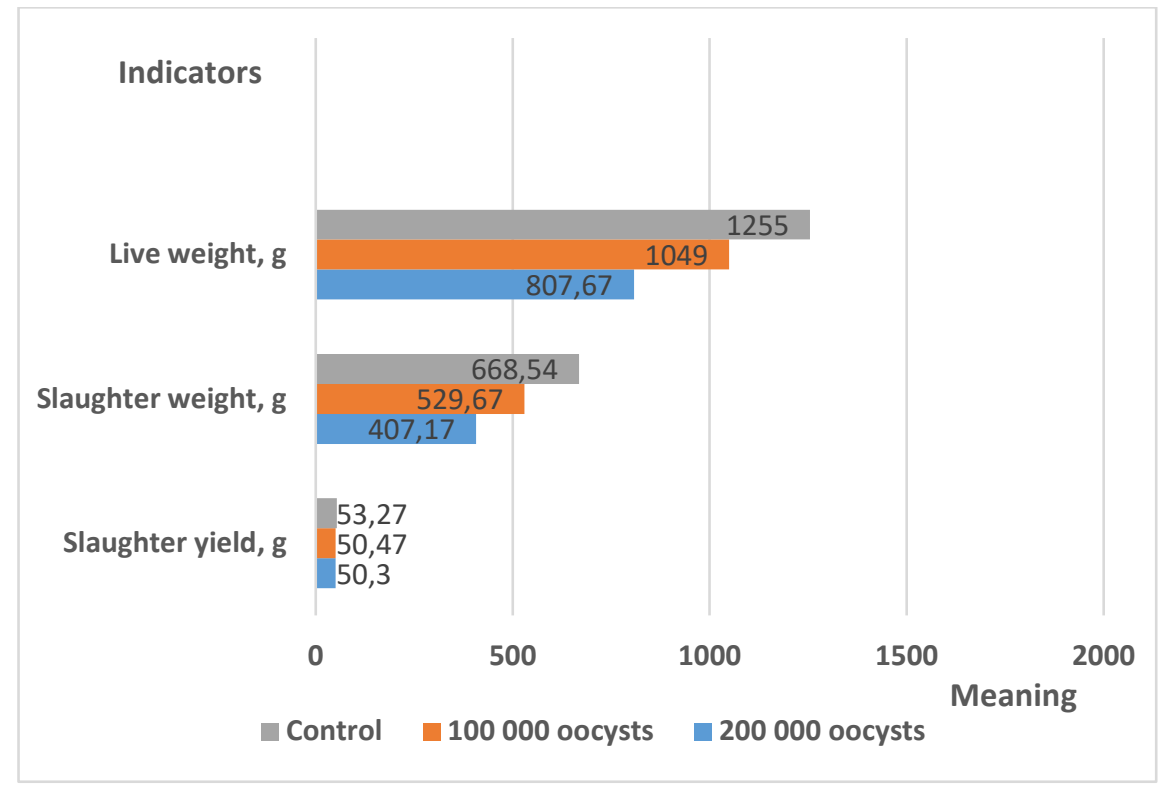

Fig. 1. Slaughter yield of carcasses of experimental animals on the 30th day after infection. A similar trend is shown by rabbits at 45 days of age (Figure 2).

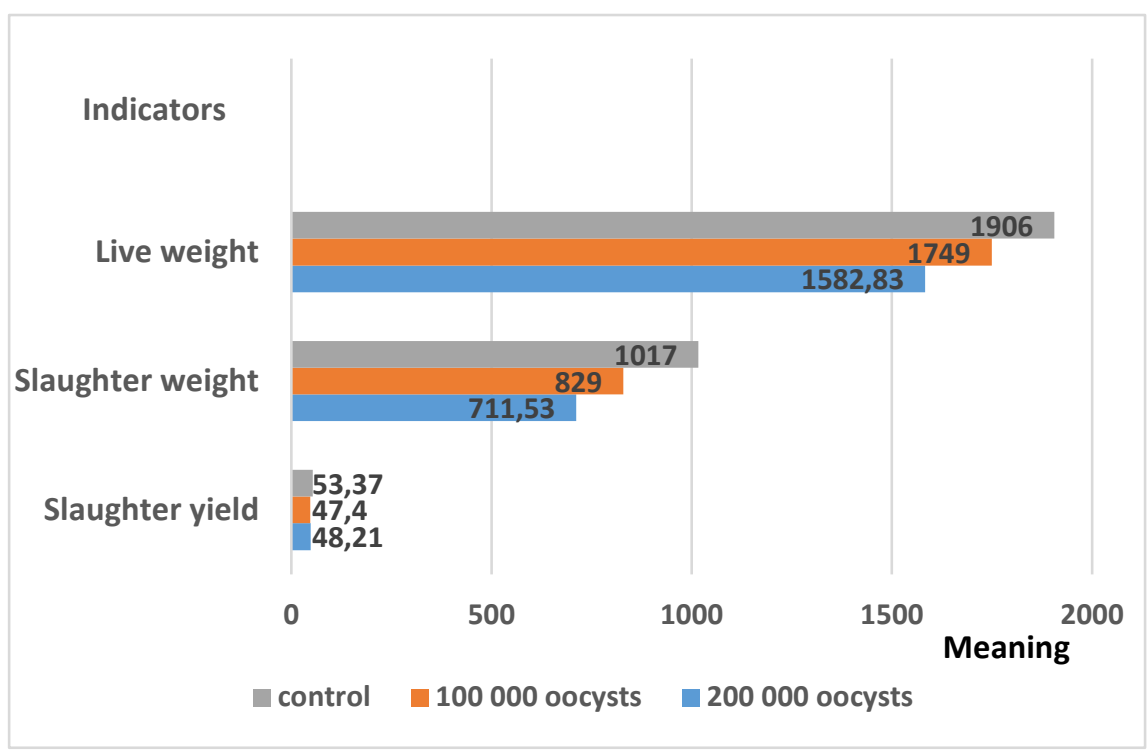

Fig. 2. Slaughter yield of carcasses of experimental animals on the 45th day after infection.

However, the decrease in the live weight of the infected groups in comparison with the control ones becomes less. In rabbits of this age group, the difference between intact rabbits and rabbits that received 200000 oocysts decreased to $16.8 \%$, and the gap between the control and the group that received an average dose of eimeria - to $8.2 \%$. In rabbits of this age group, the difference between intact rabbits and rabbits that received 200 thousand oocysts decreased to $16.8 \%$, and the gap between the control and the group that received an average dose of eimeria - to $8.2 \%$. The decrease in the slaughter yield in animals infested with 100000 oocysts as compared to the control, namely $5.16 \%$, versus $3 \%$ in the age group of 30 days, became more noticeable. However, the difference in this indicator in the groups 
infected with 100000 oocysts and 200000 oocysts becomes more noticeable and amounts to almost $1 \%$.

When considering the slaughter weight indicator, it can be seen that the most modest result is shown by the rabbits that received the most significant dose of invasion, yielding to intact animals 30\% (305.5 g), while the group infected with 100000 oocysts shows a decrease by $18 \%(188 \mathrm{~g})$ as compared to the control.

The results of measurement and comparison of the linear dimensions of the small and large intestine depending on age and dose of infection are shown in Figures 3 and 4.

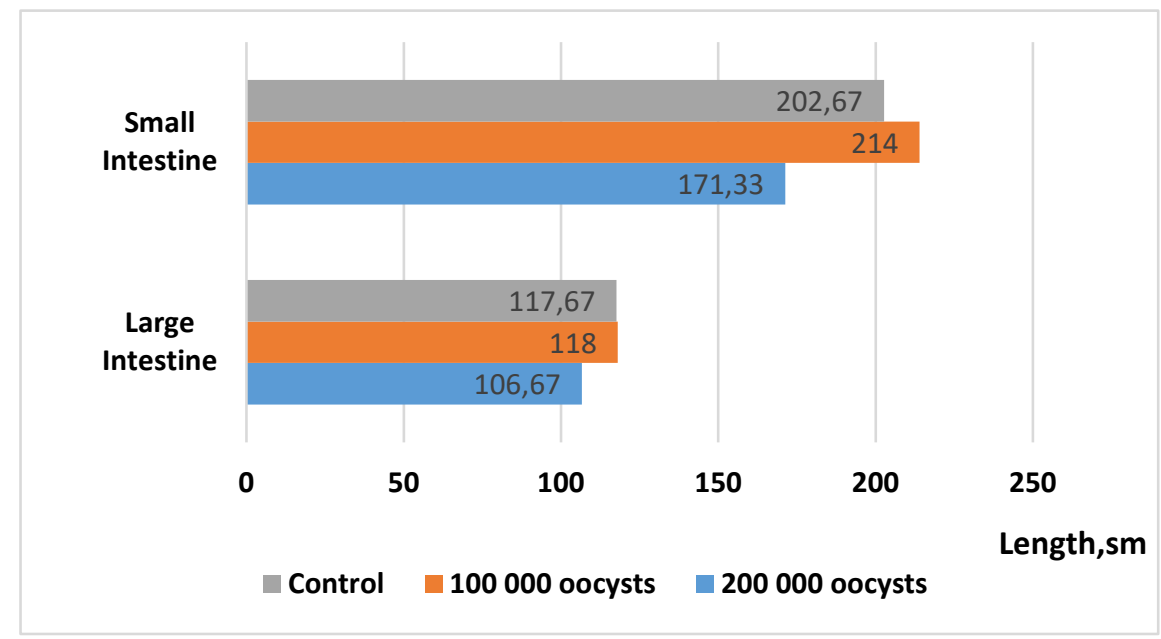

Fig. 3. Linear dimensions of the small and large intestine 30 days after infection.

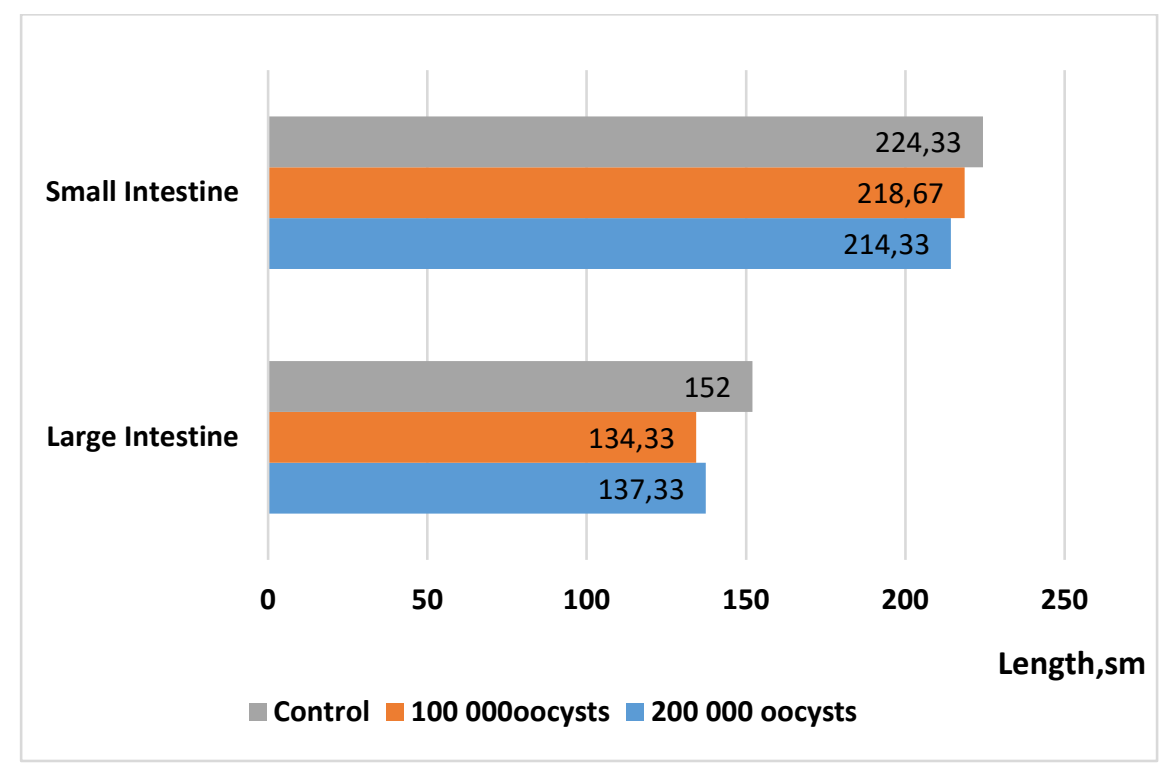

Fig. 4. Linear dimensions of the small and large intestine 45 days after infection.

It should be noted that only on the 30th day and at a low dose of infection, linear measurements of the small and large intestines overcame the same indicators in intact rabbits. The difference was only $5.6 \%$ and $0.28 \%$, respectively, but this is the only case in this study. At the same dose of invasion, but 45 days after infection, it can be seen that the size of the 
small and large intestines is smaller as compared to intact rabbits $-2.5 \%$ and $11.6 \%$ respectively.

The organoleptic assessment of the carcasses of slaughtered rabbits of different ages did not reveal any changes in the quality of meat.

Pathological studies of internal organs showed that with moderate and strong invasion intensity (100 000 and 200000 oocysts per head), regardless of the age of the hybrid rabbits, pathomorphological changes were recorded only in the small intestine, in the form of small foci of petechial and banded hemorrhages, as well as small areas of hyperemia of the serous membrane of the small intestine.

The results of a parasitological autopsy and examination of the contents of various parts of the intestine confirmed the dependence of the intensity of the invasion by eimeria from the dose of infection.

The histological examination of the small and large intestines of the invasive hybrid rabbits of the presented ages and groups showed that despite minor pathomorphological changes in the mucous membrane of the small intestine damage of the enterocytes, as well as of the villi of the single-layer prismatic banded epithelium was observed (Figure 5).

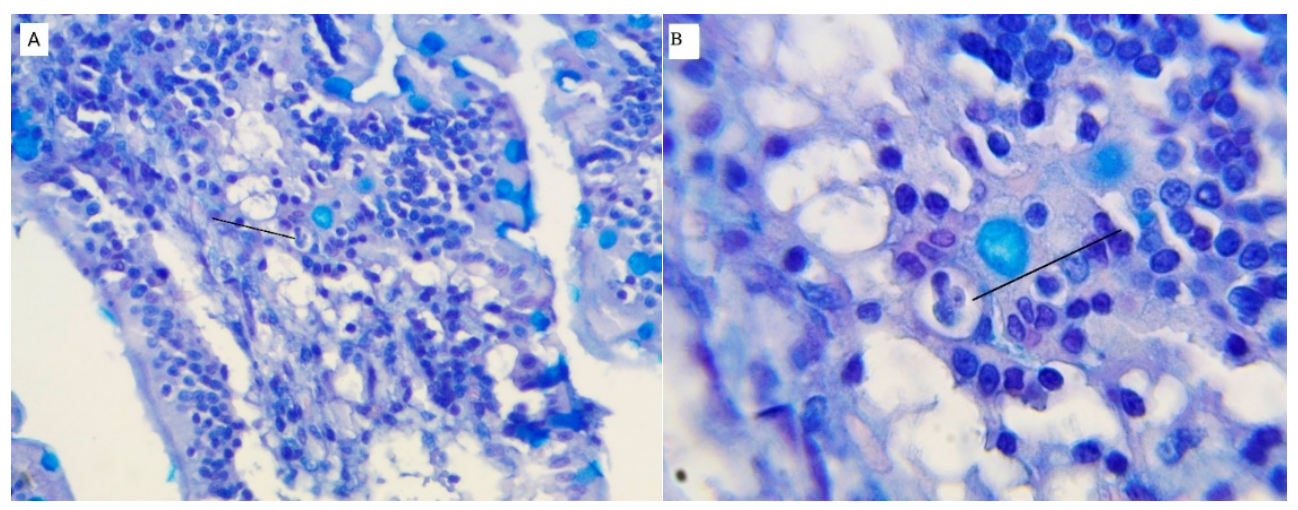

Fig. 5. Damage caused by endocytic stages of eimeria in the lamina propria of small intestine mucosa. (Coloring: alcian blue and hematoxylin Carazzi, magnification: x400 (A) and x1000 (B)).

In the thickness of the epithelial plate, intracellular stages of eimeria were found at different stages of merogony. Oval, round, or ovoid trophozoites with a pronounced basophilic nucleus inside the parasitoform vacuole were most often found in the crypts. There were signs of stromal and subepithelial edema, with extensive ruptures of the loose fibrous connective tissue of its own mucosal plate and detachment of the epithelial plate due to its necrotization.

The histological examination of the colon, liver, spleen and lymph nodes of experimental animals of all ages and doses of infection did not reveal any morphological changes in the structure of these organs.

\section{Conclusions}

Thus, the results of our study have shown that within moderate and strong infection intensity of 100000 and 200000 oocysts per head, respectively, the minimum level of weight gain and slaughter yield of carcasses was observed in rabbits slaughtered on the 30th day at a dose of infection of 200000 oocysts, at the same time despite the preservation of the retail display of the carcasses and their taste are preserved.

Our preliminary studies have shown that the degree of intensity of invasion by eimeria does not affect the linear measurements of the intestinal tube sections. 


\section{References}

1. S. El-Ashram, S.M. Aboelhadid, E.M. Abdel-Kafy, S.A. Hashem, L.N.Mahrous, E.M. Farghly, A.A. Kamel, Animals, 10(3), 537 (2020)

2. J.E. Hermansen, K. Horsted, A.G. Kongsted, Encyclopedia of Meat Sciences. Reference Work. Second Edition (2014) URL: https://www.sciencedirect.com/topics/foodscience/rabbit-meat

3. S.G. Chavhan, Lal Jangir Babu, N.V. Kurkure, N.A. Chopade, URL: https://www.researchgate.net/publication/252055448_Infectious_Diseases_of_Rabbits.

4. P.L. Long, B.J. Millard, Parasitology, 78, 239-247 (1979)

5. A. Polozowski, Wiad Parazytol, 39(1), 13-28 (1993)

6. T.K. Bhat, K.P.Jithendran, N.P. Kudare, World Rabbit Science, 4(1), 37 - 41 (1996)

7. F.L.C. Freitas, et. al., Braz. J. of Vet Path., 20, 121-126 (2011)

8. Y. Petrova, T. Georgieva, D. Zapryanova, A. Ivanov, P. Iliev, I. Kalkanov, K. Arabkercyan, Tradition and modernity in veterinary medicine, 2(5), 72-78 (2018)

9. K. Sidorenko, M. Mkrtchyan, Y. Kuznetsov, E.Klimova, Advances in Animal and Veterinary Sciences, 8 (2), 7-11 (2020)

10. G. Yin, M.U. Goraya J. Huang, X. Suo, Z. Huang, X. Liu., SpringerPlus 5, 870 (2016) URL:https:/www.researchgate.net/publication/305040736_Survey_of_coccidial_infect ion_of_rabbits_in_Sichuan_Province_Southwest_China 